\title{
Whether to Delay the Release of eBooks or Not? An Analysis of Optimal Publishing Strategies for Book Publishers
}

\section{Fan $\mathrm{Li}^{1}$, Shengli $\mathrm{Li}^{2}$ and Jiarong $\mathrm{Gu}^{3}$}

\author{
${ }^{1}$ Shenzhen University, China Center for Special Economic Zone Research, Shenzhen, China, lifan@szu.edu.cn \\ 2 Peking University, Department of Information Management, Beijing, China, lishengli@pku.edu.cn \\ ${ }^{3}$ Xi'an Jiaotong University, School of Management, Xi'an, China, alipeach@stu.xjtu.edu.cn
}

Received 3 December 2017; received in revised form 7 June 2018; accepted 11 June 2018

\begin{abstract}
The eBooks market has been growing rapidly in recent years. At the same time, evidence shows that eBooks are cannibalizing the market for physical books. To alleviate the cannibalization effect between eBooks and physical books, publishers may choose to delay the release of eBooks or physical books for a period of time. Adopting a stylized two-period model, this paper investigates three publishing strategies for a monopolistic publisher: delaying the release of eBooks, delaying the release of physical books, and releasing them simultaneously. We find that the publisher should delay the format that has significantly less market potential. Furthermore, the one that has greater market potential should be priced higher. In addition, a stronger substitution effect between eBooks and physical books favors delaying strategies. Finally, the smaller marginal cost for eBooks than physical books renders the strategy to delay the release of physical books more popular.
\end{abstract}

Keywords: eBook, Publishing strategies, Substitution effect, Cannibalization, Pricing strategies 


\section{Introduction}

In recent years, with the rapid development of the Internet and digital technology, the traditional way of reading has undergone significant changes. Since its debut in the past century, the eBook-defined as an electronic version of a printed book-has become an increasingly popular medium of reading. The release of the Kindle by Amazon in 2007 and iBooks store by Apple in 2010 further facilitated the wide acceptance of eBooks. Global eBooks revenue has grown from $\$ 1.42$ billion in 2009 to $\$ 15.87$ billion in 2016 [19].

The other side of the story, however, addresses the rising concern of the publishing industry that the demand for physical books, the counterpart to eBooks, has been cannibalized by eBooks. In essence, eBooks and physical books deliver the same content through different mediums. Most common readers will choose one of the two rather than purchasing them simultaneously. Thus, this substitution effect between eBooks and physical books leads to a cannibalization of demand for physical books by eBooks. For example, although the sale of eBooks is expected to grow, sales of most printed book categories are expected to decrease. The eBook share of total book sales is forecasted to grow to more than 25 percent in 2018, more than double the rate in 2013 [19].

From the publisher's perspective, the cannibalization effect between eBooks and physical books could potentially lead to profit loss. When facing this challenge, the publishers may adopt different publishing strategies to avoid this cannibalization effect. For example, Simon \& Schuster and Hachette announced they would delay the release of eBooks by up to four months after the release of the physical version of the same title [18] . In contrast, some publishers release the physical books after the eBooks. For example, the novel The Girl on the Train: A Novel was first available as eBook on January 13, 2015, and the physical version was not released unil August 23, 2016. Of course, the two versions can also be published simultaneously. One example is the book Killing the Rising Sun: How America Vanquished World War II Japan, which was released on September 13, 2016, in both the electronic version and as a physical book.

Thus, the publisher has three publishing strategies to choose from: delaying the release of eBooks, delaying the release of physical books, or releasing the two versions simultaneously. The publishers adopt these different strategies to alleviate the cannibalization effect between eBooks and physical books and, ultimately, to achieve optimal profit.

Which publishing strategy is optimal for the publisher is not a straightforward decision. Thus, this problem deserves a thorough analysis. Our research aims to provide guidelines on the publisher's optimal choice of publishing strategy in a monopolistic market. In particular, this paper addresses the following research questions:

Which of the three publishing strategies is optimal in terms of profitability and under what conditions?

What is the impact of the substitution effect between eBooks and physical books on the comparison between the three publishing strategies?

How should the publisher price eBooks and physical books under each publishing strategy?

We adopt a stylized two-period model to analyze the above research questions. We examine a monopolistic market where the publisher seeks to release the physical and electronic version of the same title to the market. It chooses one of the three publishing strategies to maximize its profit. Under publishing strategy 1 , the physical book and eBook are released simultaneously in the beginning of period 1 . Under strategy 2 , the release of the eBook is delayed until the beginning of period 2 . Under strategy 3 , the release of the physical book is delayed until the beginning of period 2 . The publisher determines the prices of the eBook and the physical version. Note that the eBook and physical book are considered substitutes in this model, and thus, a relatively higher price of the physical book leads to higher demand for the eBook and vice versa. The stronger the substitution effect is, the higher the cross-price elasticity is.

We find that the ratio between the market potential of the eBook and the physical book is a key factor in determining the publisher's optimal publishing strategy. When the ratio is low-that is, the eBook has a relatively lower potential market than physical book-the publisher should delay the release of the eBook to achieve the optimal profit. When this ratio is high, the publisher should delay the release of physical book. Finally, when this ratio is medium, the publisher should release the eBook and physical book simultaneously.

We further explored the optimal prices under each publishing strategy and find that higher market potential also leads to a higher optimal price. The price of the eBook is higher when it has a higher market potential than the physical book. The same occurs for the physical book. We also find that the optimal publishing strategy also leads to the highest prices for both the eBook and the physical book.

Additionally, the stronger substitution effect between eBook and physical book favors the delaying publishing strategies, either delaying the eBook or delaying the physical book. 
Finally, we find that when the marginal production costs for eBooks are significantly lower than that for physical books, the strategy to delay the release of physical books is optimal for a larger range of ratio between the market potential of the eBook and the physical book.

Our research is related to several streams of prior research. The eBook has, in essence, the same content as the physical book but is distributed through a digital channel, whereas the physical book is distributed through the traditional channel. Thus, the previous literature on dual channels sheds light on our research. Many studies focus on characterizing the conditions under which a firm should implement both physical and digital channels (e.g., [8], [11], [15], [23], [24]). Several scholars explored the pricing strategies under the setting of dual channel (e.g., [4], [16], [14]). In line with their research, we found that the optimal pricing policies for the publisher depend on the relative market potential of the eBook and the physical book.

More closely related to this research, some researchers investigated the cannibalization effects between the two channels. [2] uses an analytic model to analyze the competition between the direct channel and traditional channel. Similarly, our paper also addresses the competition between the physical book and the eBook. Using a dataset from the newspaper industry, [9] examines the cannibalization effect of the Internet channel and found that the magnitude of the cannibalization depends on whether the Internet channel mimics the traditional channel. [3] found that the introduction of an online music store did not significantly cannibalize physical record sales. [22] found significant depressing effects by the web channel on conventional television viewing and offsetting positive relationships, which reflects the substitution effect between the two channels. Based on these previous empirical results, we model the substitution between the eBook and the physical book and analyze the impact of the strength of the substitution effect.

Our research also contributes to the literature on topics related to eBooks. [13] studied when a publisher should provide an eBook along with the physical book. [1], [12], [21], [25] compared the agency selling and the traditional reselling formats in the eBook market. [5] found, counter intuitively, that delaying the eBook may lead to a large decrease in eBook sales and an increase of physical book sales. Our research verifies these empirical findings by characterizing the condition under which the publisher should not delay the eBook. Additionally, [6] proposed a quantitative model to optimize eBook retailers' free partial sampling strategy with a limited budget and a dynamic programming model to apply partial sampling over a relatively long period of time. Simulation results show the proposed models to be effective.

To the best of our knowledge, we are the first to offer guidelines to book publishers on their strategies to delay the release of eBooks or physical books. In particular, we examined the impact of the substitution effect between physical books and eBooks. In addition, we also explored book publishers' pricing strategies when different delaying strategies are adopted. Furthermore, the impact of low marginal costs for eBooks are also analyzed.

The rest of the paper is organized as follows. Section 2 sets up the analytical model and analyzes the three publishing strategies. Section 3 conducts a series of numerical analyses to reveal more managerial insights. Section 4 discusses the results and concludes the paper.

\section{Model}

In this section, we formulate the publisher's profit functions and derive the optimal profits under each publishing strategy. We then proceed to derive the conditions under which the publisher should adopt a particular publishing strategy.

\subsection{Demand Functions for the Physical Book and the eBook}

The demand functions for the physical book and the eBook are derived as follows.

We assume there is one monopolistic publisher in the market, who determines the prices of both the physical book and the eBook. The content of the physical book and the eBook are the same.

In line with traditional literature in Economics and Marketing (e.g. [9]), the demands for the eBook and the physical book are given by:

$$
\begin{gathered}
Q_{d}=A_{d}-B_{d} * P_{d}+\sigma\left(P_{t}-P_{d}\right) \\
Q_{t}=A_{t}-B_{t} * P_{t}+\sigma\left(P_{d}-P_{t}\right)
\end{gathered}
$$

$Q_{d}$ and $Q_{t}$ denote the demand for the eBook and the physical book, respectively. The demand functions do not restrict the case where a consumer buys both the eBook and the physical book. However, consumers who only purchase one version contributes to a cannibalization to the other version.

$A_{d}$ and $A_{t}$ indicate the market potential of the eBook and the physical book, i.e., the maximum possible demand. $P_{d}$ and $P_{t}$ indicate the prices of the eBook and the physical book, respectively. Note that the market potential of eBooks can be measured by the sales of eBook readers while the market potential of physical books could be obtained through 
some market research (e.g. sending surveys to potential users or predict sales from historical sales of books belong to the same author or similar topics).

$B_{d}$ and $B_{t}$ indicate the direct effect of its price change on the demand for eBook (physical book). $\sigma$ captures the substitution effect between the physical book and the eBook. The modelling of the substitution effect in our paper is similar to that in [25]. That is, a relatively lower price of eBook (physical book) leads to a higher demand for the eBook (physical book). The larger $\sigma$ is, the stronger the substitution effect is. In addition, we define $m=A_{d} / A_{t}$, the ratio of the market potential of eBook to that of physical book. If $m>1$, the eBook has greater market potential than the physical book does; otherwise, the physical book has greater market potential

\subsection{Optimal Profits under the Three Publishing Strategies}

The publisher should determine the optimal prices for the eBook and the physical book and select the optimal publishing strategy to maximize profit. As stated above, there are three publishing strategies to choose from: publishing the physical book first, publishing the eBook first, and publishing them simultaneously. We then analyze the three publishing strategies as follows.

Strategy 1. Release the physical book and the eBook simultaneously

In this case, the publisher maximizes its profit as follows:

$$
\begin{gathered}
\max _{P_{t}, P_{d}} 2 *\left[P_{d} * Q_{d}+P_{t} * Q_{t}\right] \\
\text {.t. } Q_{d} \geq 0, Q_{t} \geq 0
\end{gathered}
$$

Similar to previous literature [7], [17], the marginal costs of physical books and eBooks both are assumed to be negligible. However, this assumption is relaxed in Sec. 3.4 in which non-negligible marginal costs are included.

Solving this optimization problem leads to lemma 1.

Lemma 1. When releasing the physical book and the eBook simultaneously, the publisher should set the price of the physical book as $P_{t 1}=\frac{\sigma A_{d}+\sigma A_{t}+A_{t} B_{d}}{2\left(\sigma B_{d}+\sigma B_{t}+B_{d} B_{t}\right)}$ and the price of the eBook as $P_{d 1}=\frac{\sigma A_{d}+\sigma A_{t}+A_{d} B_{t}}{2\left(\sigma B_{d}+\sigma B_{t}+B_{d} B_{t}\right)}$. The publisher achieves a total profit of $\pi_{a 1}=\frac{2 \sigma A_{d} A_{t}+A_{t}^{2}\left(\sigma+B_{d}\right)+A_{d}^{2}\left(\sigma+B_{t}\right)}{2\left(\sigma B_{t}+B_{d}\left(\sigma+B_{t}\right)\right)}$, including a profit of $\pi_{t 1}=\frac{A_{t}\left(\sigma A_{d}+A_{t}\left(\sigma+B_{d}\right)\right)}{2\left(\sigma B_{t}+B_{d}\left(\sigma+B_{t}\right)\right)}$ from the physical book and a profit of $\pi_{d 1}=\frac{A_{d}\left(\sigma A_{t}+A_{d}\left(\sigma+B_{t}\right)\right)}{2\left(\sigma B_{t}+B_{d}\left(\sigma+B_{t}\right)\right)}$ from the eBook.

Proof: Please see Appendix A for the proof of Lemma 1.

Strategy 2. Delay release of the eBook

When delaying release of the eBook, the publisher only releases the physical book in period 1 . Thus, there is no substitution between physical book and eBook. The demand for the physical book in period 1 is given by:

$$
Q_{f 1}=A_{t}-B_{t} * P_{t}
$$

Thus, the publisher's profit in period 1 is given by $P_{t} *\left(A_{t}-B_{t} * P_{t}\right)$.

In period 2, the publisher releases the eBook to the market and the demand for the physical book and the eBook are given by Eqs. (1) and (2). The profit in period 2 thus equals $P_{d} * Q_{d}+P_{t} * Q_{t}$. The publisher maximizes the sum of profits in period 1 and period 2 by solving the following optimization problem:

$$
\begin{gathered}
\max _{P_{t} P_{d}} P_{t} * Q_{f 1}+P_{d} * Q_{d}+P_{t} * Q_{t} \\
\text { s.t. } Q_{d} \geq 0, Q_{t} \geq 0, Q_{f 1} \geq 0
\end{gathered}
$$

We then obtain lemma 2 by solving the above optimization problem.

Lemma 2. When the release of the eBook is delayed, the publisher should set the price of the physical book as $P_{t 2}=$

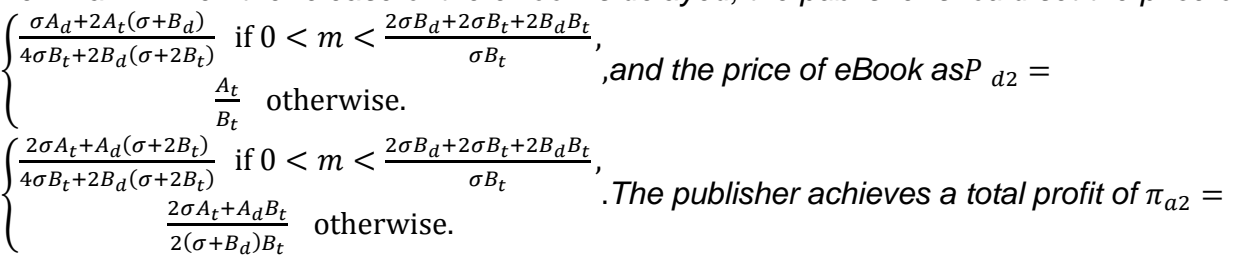




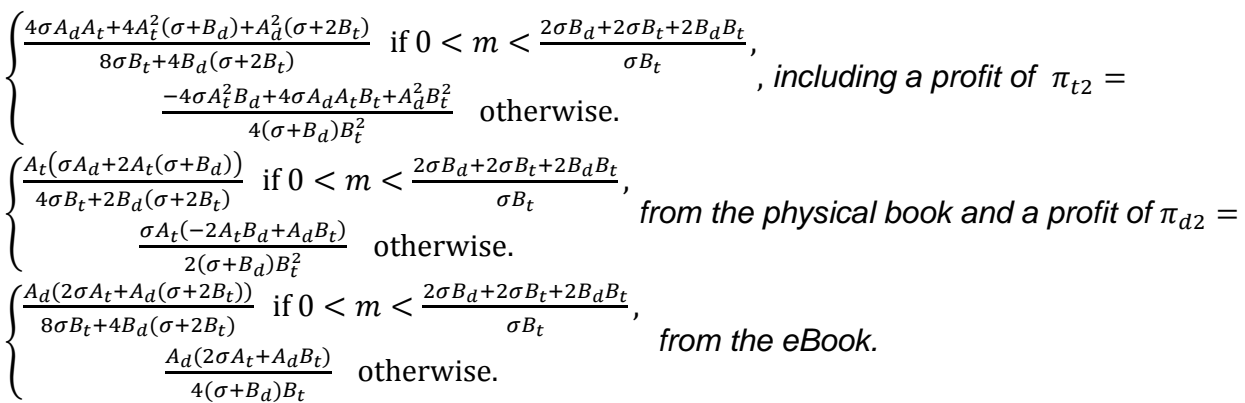

Proof: Please see Appendix A for the proof of Lemma 2.

Strategy 3. Delay release of the physical book

The publisher delays of the release of physical book to the period 2. Thus, only the eBook is on the market in period 1 , and the demand for eBook demand in that period is given by

$$
Q_{f 2}=A_{d}-B_{d} * P_{d}
$$

The first period profit equals $P_{d} *\left(A_{d}-B_{d} * P_{d}\right)$. In the second period, the physical book is released and the demand for the eBook and the physical book are given by Eqs. (1) and (2), respectively. To maximize the total profit across the two periods, the publisher faces the following optimization problem:

$$
\begin{gathered}
\max _{P_{t} P_{d}} P_{d} * Q_{f 2}+P_{d} * Q_{d}+P_{t} * Q_{t} \\
\text { s.t. } Q_{d} \geq 0, Q_{t} \geq 0, Q_{f 2} \geq 0
\end{gathered}
$$

Solving the optimization problem yields the following lemma:

Lemma 3. When delaying the release of the physical book, the publisher should set the price of the physical book as

$P_{t 3}=\left\{\begin{array}{c}\frac{2 \sigma A_{d}+A_{t} B_{d}}{2 B_{d}\left(\sigma+B_{t}\right)} \text { if } 0<m<\frac{\sigma B_{d}}{2 \sigma B_{d}+2 \sigma B_{t}+2 B_{d} B_{t}}, \\ \frac{2 \sigma A_{d}+A_{t}\left(\sigma+2 B_{d}\right)}{2 \sigma B_{t}+4 B_{d}\left(\sigma+B_{t}\right)} \text { otherwise. }\end{array}\right.$, and the price of the eBook as $P_{d 3}=\left\{\begin{array}{c}\frac{A_{d}}{B_{d}} \text { if } 0<m<\frac{\sigma B_{d}}{2 \sigma B_{d}+2 \sigma B_{t}+2 B_{d} B_{t}}, \\ \frac{\sigma A_{t}+2 A_{d}\left(\sigma+B_{t}\right)}{2 \sigma B_{t}+4 B_{d}\left(\sigma+B_{t}\right)} \text { otherwise. The }\end{array}\right.$ publisher achieves a total profit of $\pi_{a 3}=\left\{\begin{array}{c}\frac{4 \sigma A_{d} A_{t} B_{d}+A_{t}^{2} B_{d}^{2}-4 \sigma A_{d}^{2} B_{t}}{4 B_{d}^{2}\left(\sigma+B_{t}\right)} \text { if } 0<m<\frac{\sigma B_{d}}{2 \sigma B_{d}+2 \sigma B_{t}+2 B_{d} B_{t}}, \\ \frac{4 \sigma A_{d} A_{t}+A_{t}^{2}\left(\sigma+2 B_{d}\right)+4 A_{d}^{2}\left(\sigma+B_{t}\right)}{4 \sigma B_{t}+8 B_{d}\left(\sigma+B_{t}\right)} \text { otherwise. }\end{array}\right.$ including a profit of $\pi_{t 3}=$ $\left\{\begin{array}{cl}\frac{A_{t}\left(2 \sigma A_{d}+A_{t} B_{d}\right)}{4 B_{d}\left(\sigma+B_{t}\right)} \text { if } 0<m<\frac{\sigma B_{d}}{2 \sigma B_{d}+2 \sigma B_{t}+2 B_{d} B_{t}}, & \text { from the physical book and a profit of } \pi_{d 3}= \\ \frac{A_{t}\left(2 \sigma A_{d}+A_{t}\left(\sigma+2 B_{d}\right)\right)}{4 \sigma B_{t}+8 B_{d}\left(\sigma+B_{t}\right)} & \text { otherwise. }\end{array}\right.$ $\left\{\begin{array}{c}\frac{\sigma A_{d}\left(A_{t} B_{d}-2 A_{d} B_{t}\right)}{2 B_{d}^{2}\left(\sigma+B_{t}\right)} \text { if } 0<m<\frac{\sigma B_{d}}{2 \sigma B_{d}+2 \sigma B_{t}+2 B_{d} B_{t}}, \text { from the eBook. } \\ \frac{A_{d}\left(\sigma A_{t}+2 A_{d}\left(\sigma+B_{t}\right)\right)}{2 \sigma B_{t}+4 B_{d}\left(\sigma+B_{t}\right)} \text { otherwise. }\end{array}\right.$

Proof: Please see Appendix A for the proof of Lemma 3.

\subsection{Comparison of the Three Strategies}

We now compare the optimal profits derived under the three strategies and determine under what conditions each strategy is optimal. Due to the complexity, a direct comparison between the above optimal profit expressions provides no analytical insights. Therefore, without loss of generality, we assume $B_{t}=1$ and $B_{d}=1$. That is, demand for both the eBook and the physical book is unit elastic. The comparison leads to the following proposition:

Proposition 1. let $m=A_{d} / A_{t}, m_{1}=-\frac{2\left(\sigma+\sigma^{2}\right)}{2+5 \sigma+4 \sigma^{2}}+\sqrt{2} \sqrt{\frac{2 \sigma+9 \sigma^{2}+13 \sigma^{3}+6 \sigma^{4}}{\left(2+5 \sigma+4 \sigma^{2}\right)^{2}}}, m_{2}=1+\frac{\sqrt{2+7 \sigma+6 \sigma^{2} / \sigma(1+\sigma)}}{\sqrt{2}}$. If $m<m_{1}$, the publisher's optimal strategy is to delay the eBook for one period; if $m>m_{2}$, the publisher prefers to delay the physical book for one period; finally, if $m_{1} \leq m \leq m_{2}$, the publisher releases the two versions simultaneously to achieve the highest profit.

Proof: Please see Appendix A for the proof of Proposition 1. 
Proposition 1 reveals that the ratio of the market potential of the eBook to that of the physical book is a key factor influencing the optimal publishing strategy. When this ratio is low, the publisher should delay the release of the eBook. On the other hand, the publisher should delay the release of the physical book when this ratio is high. Finally, when this ratio is medium, the publisher should release the two versions simultaneously. Figure 1 illustrates the results of Proposition 1.

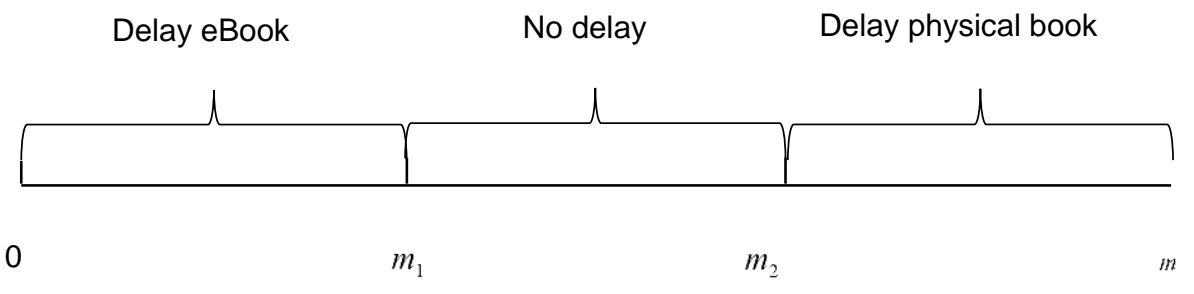

Figure 1: The optimal publishing strategy

\section{Numerical Analysis}

We explore the influences of several key factors in the comparison between the three publishing strategies in this section.

\subsection{The impact of the Ratio between the Market Potentials}

Proposition 1 reveals the impact of the ratio between the market potential of the eBook and that of the physical book on the publisher's optimal strategy. Here, we use a numerical example to illustrate how the optimal profits are achieved under each publishing strategy change with respect to this ratio. Let $B_{t}=1, B_{d}=1$ and $\sigma=0.3$, and we obtain Figure 2.

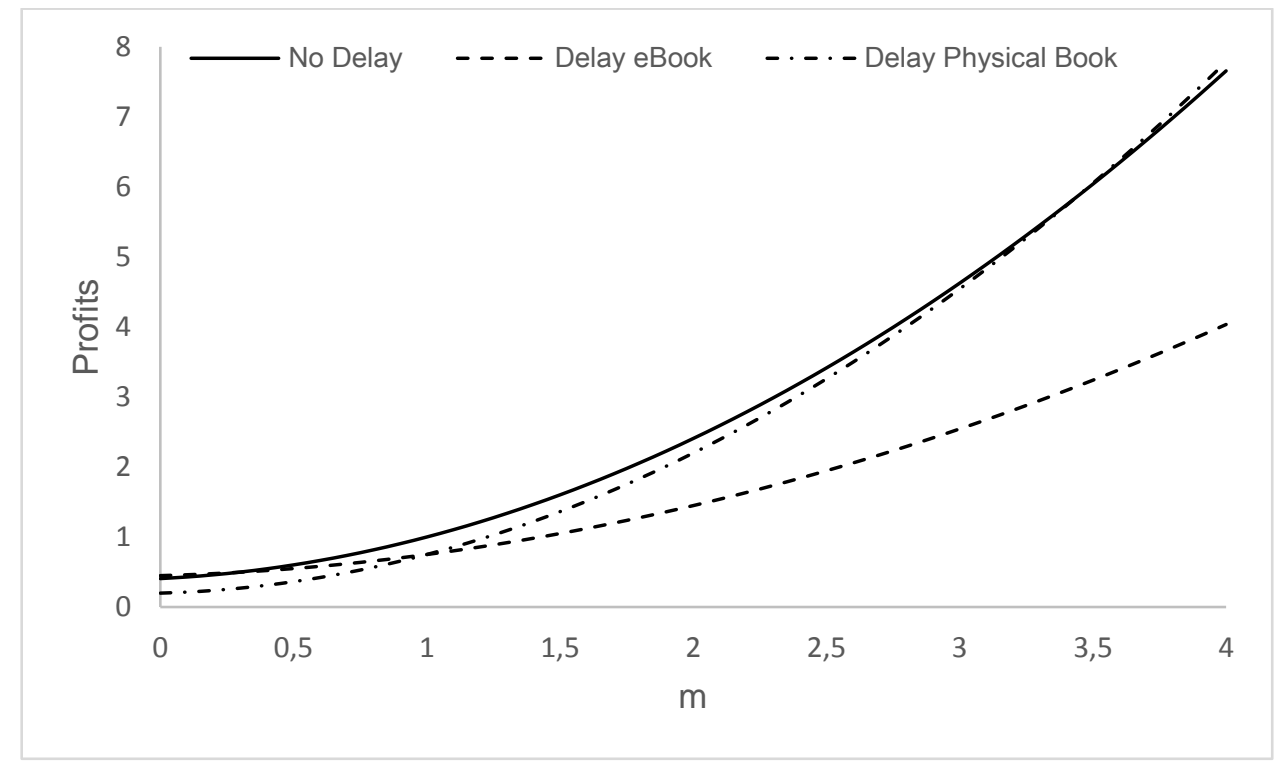

Figure 2: Comparison between the three publishing strategies

Figure 2 shows that when $m=A_{d} / A_{t}$ is small (smaller than 0.3), delaying the release of the eBook leads to the highest profit. When $m$ is large (greater than 3.5), the publisher achieves the highest profit when delaying release of the physical book. Finally, when $m$ is in the middle range (within the interval $[0.3,3.5]$ ), the publisher's optimal strategy is to release the two versions simultaneously.

Next, we illustrate the profits gained from the physical book and the eBook, respectively, when the two versions are released simultaneously. This helps us to understand why the publisher needs to delay releasing the eBook (or the physical book) under certain conditions. The numerical results are presented in Figure 3. 


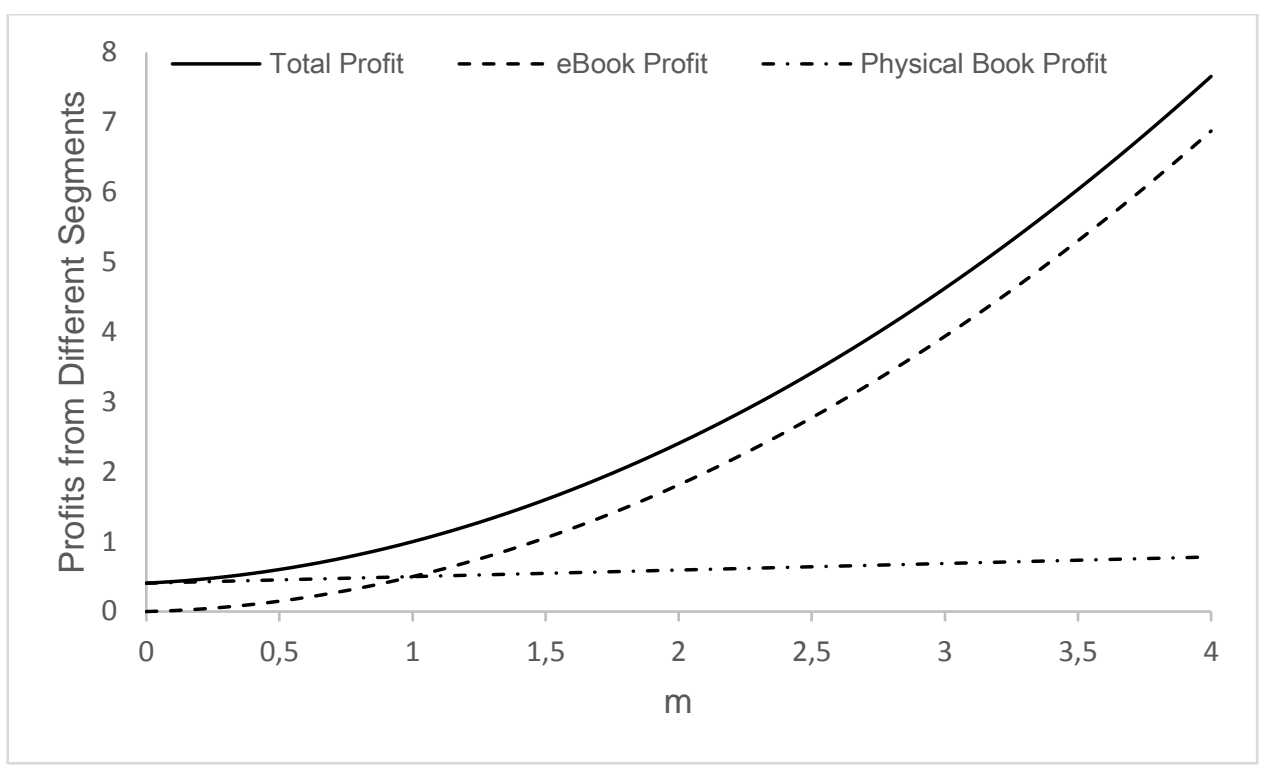

Figure 3: Profits gained from different segments (in the case of no delay)

As Figure 3 shows, when $m$ is small, the profit gained from the eBook market is much lower than that from the physical book market. Thus, the best strategy in this case is to give up the market for eBook for one period (that is, to delay the eBook for one period) to avoid the cannibalization effect between the eBook and the physical book. This is why we observe that delaying the release of the eBook is optimal when $m$ is smaller than a certain threshold value $(0.3$ in Figure 3). When $m=1$, the profit from eBook and the profit from the physical book are identical. This can be explained by the same market power between two types of books. When $m$ becomes larger, the profit gained from the eBook market grows at a much higher rate than that from the physical book market. When $m$ falls into the medium range, the profits gained from the eBook and the physical book are comparable to each other; thus, the publisher finds that it is not optimal to delay the release of either the eBook or the physical book. Finally, when $m$ becomes large enough, the profit gained from the eBook significantly outweighs that gained from the physical book, and therefore, it is optimal to delay the release of the physical book.

\subsection{The Optimal Prices of the eBook and the Physical Book}

The figure below compares the prices of the eBook and the physical book under each of the three publishing strategies.

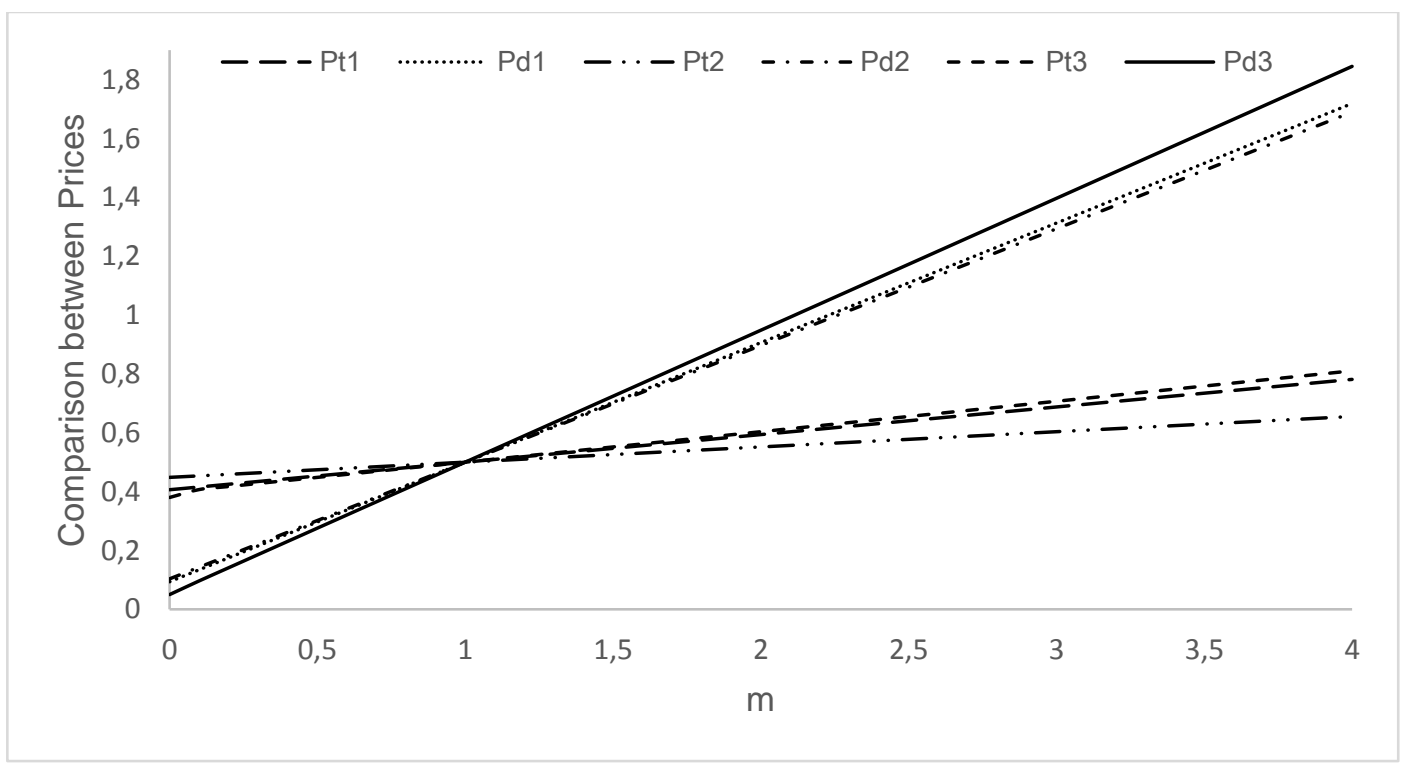

Figure 4: Prices of the eBook and the physical book under the different publishing strategies

In Figure 4, $P_{t 1}$ and $P_{d 1}$ are the prices under strategy 1 (no delay); $P_{t 2}$ and $P_{d 2}$ are the prices under strategy 2 (delay eBook); and $P_{t 3}$ and $P_{d 3}$ are the prices under strategy 3 (delay physical book). Figure 4 reveals that when $m=A_{d} / A_{t}$ is less than 1 (i.e., $A_{d}<A_{t}$ ), the prices of the physical book are always greater than those of the eBook under each 
publishing strategy. In other words, the publisher charges a higher price for the physical book when the market potential of the physical book is higher than that of the eBook. On the other hand, the price of the eBook is higher when the market potential of the eBook is higher.

Furthermore, we observe that when $m=A_{d} / A_{t}$ is greater than 1 , the price of both the eBook and the physical book are greatest under strategy 3 (delay physical book), and smallest under strategy 2 (delay eBook). On the other hand, when $m=A_{d} / A_{t}$ is less than 1, the price of both the eBook and the physical book are greatest under strategy 2 (delay eBook) and smallest under strategy 3 (delay physical book). Recall that when $m$ is small, the publisher should delay release of the eBook. We now observe that by delaying the eBook, the publisher can charge the highest price for the eBook and the physical book. In other words, delaying the eBook when $m$ is small allows the publisher to charge higher prices for both the eBook and the physical book, thereby obtaining optimal profit. When $m$ is large, the managerial implication is similar.

\subsection{The Impact of the Substitution Effect}

We now investigate the impact of the substitution effect. In particular, we illustrate how the threshold values of $m\left(m_{1}\right.$ and $m_{2}$ in Proposition 1) change with respect to $\sigma$. Let $B_{t}=1, B_{d}=1$, and we obtain Figure 5.

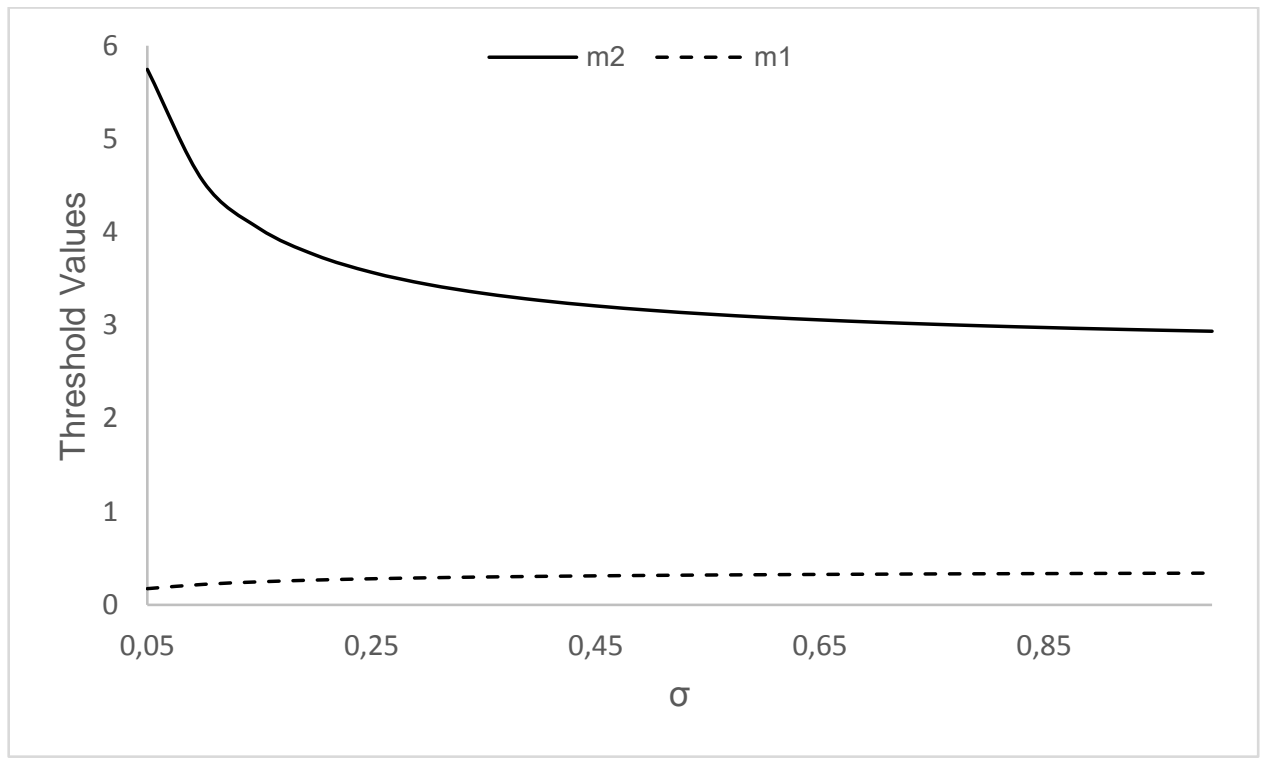

Figure 5: The impact of the substitution effect

As Figure 5 indicates, when the substitution effect becomes stronger, $m_{2}$ gets smaller and $m_{1}$ becomes larger, leading to a narrower range of $m$, where the strategy to release the two versions simultaneously is optimal. The managerial implication here is that a stronger substitution effect between the eBook and the physical book leads to fiercer competition between the two versions, which in turn causes a higher degree of demand cannibalization between them. Thus, the strategy of delaying the eBook or the physical book to avoid face-to-face competition between the two becomes more favorable when there is a strong substitution effect.

\subsection{Non-Negligible Marginal Costs}

The previous analysis assumed that the marginal costs of physical books and eBooks are negligible. This section explores the case when non-negligible marginal costs exist.

Let $c_{t}$ and $c_{d}$ denote the marginal cost of producing and distributing one copy of physical book and eBook, respectively.

The profit function of the publisher under Strategy 1 thus is given by $2 *\left[\left(P_{d}-c_{d}\right) * Q_{d}+\left(P_{t}-c_{t}\right) * Q_{t}\right]$. The profit function of the publisher when it adopts strategy 2 is given by $\left(P_{t}-c_{t}\right) * Q_{f 1}+\left(P_{d}-c_{d}\right) * Q_{d}+\left(P_{t}-c_{t}\right) * Q_{t}$. Finally, the profit function under Strategy 3 is $\left(P_{d}-c_{d}\right) * Q_{f 2}+\left(P_{d}-c_{d}\right) * Q_{d}+\left(P_{t}-c_{t}\right) * Q_{t}$.

The demand functions remain the same as in Sec. 2.1

We then conducted a numerical comparison between the profits achieved under the three strategies, considering nonnegligible marginal costs. 
Let $B_{t}=1, B_{d}=1, \sigma=0.3, c_{t}=0.15$ and $c_{d}=0.05$, we obtain Figure 6 . Note that the parameter values are chosen to reflect the conventional wisdom that the marginal cost of eBook is smaller than that of physical book [21].

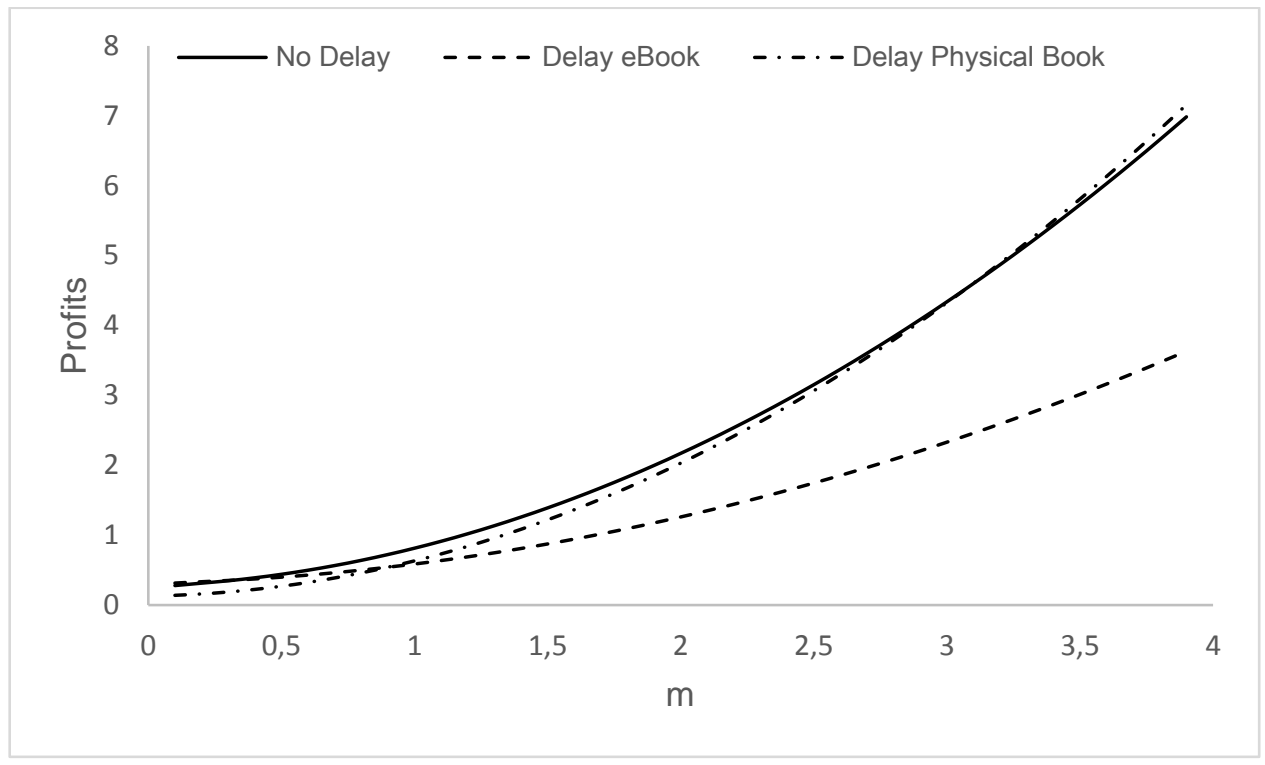

Figure 6: The comparison when non-negligible marginal costs

Firstly, comparing Figure 6 to Figure 2, we observe that the pattern of Figure 6 remains similar to that of Figure 2. It still shows that when $m=A_{d} / A_{t}$ is small, the publisher's best strategy is to delay the release of the eBook; When $m$ is large, the publisher achieves the highest profit when delaying release of the physical book; When $m$ is in the middle range, the publisher should release the two versions simultaneously. This shows the robustness of our analysis in Section 2 .

Additionally, we observe that the threshold value of $m_{2}$ shifts leftward from 3.5 in Figure 2 to approximately 3.0 in Figure 6 . This shows that the strategy of delaying the release of the physical book becomes superior for a larger range of $m$. The intuition is that when the marginal cost of the eBook is lower than that of the physical book, the eBook becomes more competitive and therefore delaying the release of the physical book becomes a profitable option in more cases.

\section{Conclusion}

With the rapid development of digital technologies, especially the widespread use of portable e-reading devices, such as Kindle and iPad, more and more readers choose to read books electronically. Responding to this market trend, current publishers typically publish both a physical and an electronic version of the same title. However, doing so may lead to cannibalization between the physical book and the eBook. The publisher may choose to delay the release of the physical book or the eBook to alleviate the cannibalization effect between them. In this paper, we develop a stylized two-period model to explore three publishing strategies: delaying release of the eBook, delaying release of the physical book, and releasing them simultaneously.

This paper has several interesting findings. First, we find that the ratio between the market potential of the eBook and the physical book is a crucial factor impacting the publisher's optimal publishing strategy. When this ratio is low enoughi.e., when the eBook has a relatively lower market potential-the publisher's best strategy is to delay the release of the eBook. When this ratio is high enough, on the other hand, the optimal strategy is to delay release of the physical book. When this ratio is medium-that is, the market potential of the eBook and the physical book are comparable-the publisher should release them simultaneously. This finding explains the reality well. When the eBook first came into the market, the number people who owned eBook readers was small, and thus the market potential for the eBook was also lower. Therefore, it was common to delay release of the eBook at that time. Today, however, with the widespread of eBook readers, the market potential of the eBook increases and we observe that it is common to release the two versions simultaneously or even delay the physical book.

We also find that the version (physical or electronic) having greater market potential also sets a higher optimal price. For example, the price of the physical book is higher when it has greater market potential than the eBook. This may explain the price changes of eBooks in recent years. When eBooks first came into the market, they were usually sold at significantly lower prices than physical books (such as $\$ 9.99$ per book). Currently, with the increase in ownership of eBook readers, we observe that eBooks are usually sold at higher prices than they were before. 
We also find that the optimal publishing strategy also leads to the highest prices of the eBook and the physical book, compared with the other two strategies. In other words, delaying the eBook (or the physical book) under certain conditions allows the publisher to charge a higher price and thus lead to optimal profit.

Additionally, our results reveal that a stronger substitution effect favors the delaying publishing strategies, either delaying the eBook or the physical book. The strength of the substitution effect between the eBook and the physical book might be different for different categories of books. For example, students prefer a printed textbook over etextbook, indicating a weak substitution effect for textbooks [20]. Therefore, for different categories of books, the publisher may need to implement different publishing strategies.

Marginal costs for eBooks are claimed to be lower than that for physical books [21]. We find that this fact renders the eBook becomes more competitive and the strategy to delay the release of physical books more popular.

This paper has several limitations. First, we do not consider the sales of eBook reading devices (e.g., Kindle, iPad) in our model. Second, we do not incorporate the wholesale and agency selling of eBooks in our model. Addressing these two issues in the future research may lead to additional managerial insights.

\section{Acknowledgement}

This work is supported by the National Natural Science Foundation of China $(71402134,71572138$, 71571140, 71473191, 91546119, and 71702109). ${ }^{2}$ Corresponding author.

\section{References}

[1] V. Abhishek, K. Jerath and Z. J. Zhang, Agency selling or reselling? Channel structures in electronic retailing, Management Science, vol. 62, no. 8, pp. 2259-2280, 2016.

[2] S. Balasubramanian, Mail versus mall: A strategic analysis of competition between direct marketers and conventional retailers, Marketing Science, vol. 17, no. 3, pp. 181-195, 1998.

[3] E. Biyalogorsky and P. Naik, Clicks and mortar: The effect of on-line activities on off-line sales, Marketing Letters, vol. 14, no. 1, pp. 21-32, 2003.

[4] K. Cattani, W. Gilland, H. S. Heese and J. Swaminathan, Boiling frogs: Pricing strategies for a manufacturer adding a direct channel that competes with the traditional channel, Production and Operations Management, vol. 15, no. 1, pp. 40-56, 2006.

[5] H. Chen, Y.J. Hu and M.D. Smith. (2011, November) The impact of eBook distribution on print sales: Analysis of a natural experiment, management science. SSRN. [Online]. Available: https://papers.ssrn.com/sol3/papers. cfm?abstract id=1966115.

[6] L. Chen, A quantitative analysis on e-books sampling optimization, Journal of Theoretical and Applied Electronic Commerce Research, vol. 12, no. 1, pp. 68-76, 2017.

[7] H.K. Cheng, S. Li and Y. Liu, Optimal software free trial strategy: Limited version, time-locked or hybrid? Production and Operations Management, vol. 24, no. 3, pp. 504-517, 2015.

[8] W.K. Chiang, D. Chhajed and J.D. Hess, Direct marketing, indirect profits: A strategic analysis of dual-channel supply-chain design, Management Science, vol. 49, no. 1, pp. 1-20, 2003.

[9] P. Desai and D. Purohit, Competition in durable goods market: The strategic consequences of leasing and selling, Marketing Science, vol. 18, no. 1, pp. 42-58, 1999.

[10] B. Deleersnyder, I. Geyskens, K. Gielens, and M. GDekimpe, How cannibalistic is the Internet channel? A study of the newspaper industry in the United Kingdom and The Netherlands, International Journal of Research in Marketing, vol. 19, no. 4, pp. 337-348, 2002.

[11] A. Dumrongsiri, M. Fan, A. Jain, and K. Moinzadeh, A supply chain model with direct and retail channels, European Journal of Operational Research, vol. 187, no. 3, pp.691-718, 2008.

[12] L. Hao and M. Fan, An analysis of pricing models in the electronic book market, MIS Quarterly, vol. 38, no. 4, pp. 1017-1032, 2014.

[13] G. Hua, T.C.E. Cheng and S. Wang, Electronic books: To e or not to e? A strategic analysis of distribution channel choices of publishers, International Journal of Production Economics, vol. 129, no. 2) pp. 338-346, 2011.

[14] G. Hua, S. Wang and T.C.E. Cheng, Price and lead time decisions in dual-channel supply chains, European Journal of Operational Research, vol. 205, no. 1, pp. 113-126, 2011.

[15] W. Huang and J.M. Swaminathan, Introduction of a second channel: Implications for pricing and profits, European Journal of Operational Research, vol. 194, no. 1, pp. 258-279, 2009.

[16] H. Kurata, D.Q. Yao and J.J. Liu, Pricing policies under direct vs. indirect channel competition and national vs. store brand competition, European Journal of Operational Research, vol. 180, no. 1, pp. 262-281, 2007.

[17] S. Li, H.K. Cheng, Y. Duan and Y. Yang, A study of enterprise software licensing models, Journal of management information systems, vol. 34, no. 1, pp. 177-205, 2017.

[18] R. Maher. (2017, March) Two major publishers delay e-book celease dates. Business Insider. [Online]. Available: http://www.businessinsider.com/two-major-publishers-delay-e-book-release-dates-2009-12.

[19] Statista. (2017, March) Statistics and facts about e-books. Statista. [Online]. Available: https://www.statista.com/ topics/1474/e-books/. 
[20] T. Tan. (2017, March) College students still prefer print textbooks. Publishers Weekly. [Online]. Available: http://www.publishersweekly.com/pw/by-topic/digital/content-and-e-books/article/63225-college-students-prefera-mix-of-print-and-digital-textbooks.html.

[21] Y. Tan and J. Carrillo, Strategic analysis of the agency model for digital goods, Production and Operations Management, vol. 26, no. 4, pp. 724-741, 2017.

[22] J. Waldfogel, Lost on the web: Does web distribution stimulate or depress television viewing? Information Economics and Policy, vol. 21, no. 2, pp.158-168, 2009.

[23] C.J. Wang, Y.J. Chen and C.C. Wu, Advertising competition and industry channel structure, Marketing Letters, vol. 22, no. 1, pp. 79-99, 2011.

[24] Y. Xia, T. Xiao and G.P. Zhang, Distribution channel strategies for a manufacturer with complementary products, Decision Sciences, vol. 44, no. 1, pp.39-56, 2013.

[25] C. Zhu and Z. Yao, Comparison between the agency and wholesale model under the e-book duopoly market, Electronic Commerce Research, vol. 18, no. 2, pp. 313-337, 2018. 


\section{Appendix A: Proof of Lemmas Propositions}

\section{Proof of Lemma 1}

According to the publisher's profit maximization problem in Eq. (3), we write the Lagrange equation as follows.

$L=2\left(P_{d} * Q_{d}+P_{t} * Q_{t}\right)+\lambda_{1}\left(A_{d}-B_{d} * P_{d}+\sigma\left(P_{t}-P_{d}\right)\right)+\lambda_{2}\left(A_{t}-B_{t} * P_{t}+\sigma\left(P_{d}-P_{t}\right)\right)$. There are four cases to discuss depending on the values of $\lambda_{1}$ and $\lambda_{2}$.

In the first case, $\lambda_{1}=0, \lambda_{2}=0$. By $\frac{\partial L}{\partial P_{d}}=0, \frac{\partial L}{\partial P_{t}}=0$, we obtain that $P_{t}=\frac{\sigma A_{d}+\sigma A_{t}+A_{t} B_{d}}{2\left(\sigma B_{d}+\sigma B_{t}+B_{d} B_{t}\right)}, P_{d}=\frac{\sigma A_{d}+\sigma A_{t}+A_{d} B_{t}}{2\left(\sigma B_{d}+\sigma B_{t}+B_{d} B_{t}\right)}$. Plugging $P_{d}, P_{t}$ into the demand function, we obtain that $Q_{d} \geq 0, Q_{t} \geq 0$. Plugging $P_{d}, P_{t}$ into the profit function, we obtain $\pi^{*}$.

In the second case, $\lambda_{1} \neq 0, \lambda_{2}=0$. By $\frac{\partial L}{\partial P_{d}}=0, \frac{\partial L}{\partial P_{t}}=0, \frac{\partial L}{\partial \lambda_{1}}=0$, we obtain that $\lambda_{1}=-\frac{2 A_{d}}{\sigma+B_{d}}<0$. Thus, this case is infeasible.

In the third case, $\lambda_{1}=0, \lambda_{2} \neq 0$. By $\frac{\partial L}{\partial P_{d}}=0, \frac{\partial L}{\partial P_{t}}=0, \frac{\partial L}{\partial \lambda_{2}}=0$, we obtain that $\lambda_{2}=-\frac{2 A_{t}}{\sigma+B_{t}}<0$. Thus this case is infeasible.

In the fourth case, $\lambda_{1} \neq 0, \lambda_{2} \neq 0$. By $\frac{\partial L}{\partial P_{d}}=0, \frac{\partial L}{\partial P_{t}}=0, \frac{\partial L}{\partial \lambda_{1}}=0, \frac{\partial L}{\partial \lambda_{2}}=0$, we obtain no solutions.

In sum, the optimal solution is obtained as given by Lemma 1.

\section{Proof of Lemma 2}

To solve the publisher's decision problem under the strategy 2, we write the Lagrange equation as follows.

$L=\left(P_{d} * Q_{d}+P_{t} * Q_{t}+P_{t} * Q_{f 1}+\lambda_{1}\left(A_{d}-B_{d} * P_{d}+\sigma\left(P_{t}-P_{d}\right)\right)+\lambda_{2}\left(A_{t}-B_{t} * P_{t}+\sigma\left(P_{d}-P_{t}\right)\right)+\lambda_{3}\left(A_{t}-B_{t} * P_{t}\right)\right.$

There are eight cases to discuss depending on the values of $\lambda_{1}, \lambda_{2}$ and $\lambda_{3}$.

In the first case, $\lambda_{1}=0, \lambda_{2}=0, \lambda_{3}=0$. By $\frac{\partial L}{\partial P_{d}}=0, \frac{\partial L}{\partial P_{t}}=0$, we obtain that $P_{t}=\frac{\sigma A_{d}+2 \sigma A_{t}+2 A_{t} B_{d}}{2\left(\sigma B_{d}+2 \sigma B_{t}+2 B_{d} B_{t}\right)}, P_{d}=\frac{\sigma A_{d}+2 \sigma A_{t}+2 A_{d} B_{t}}{2\left(\sigma B_{d}+2 \sigma B_{t}+2 B_{d} B_{t}\right)}$. Plugging $P_{d}, P_{t}$ into the demand function, we can obtain that $Q_{d} \geq 0, Q_{t} \geq 0$. Plugging into the constraints $Q_{f} \geq 0$ and let $m=A_{d} / A_{t}$, we obtain the condition $0<m<\frac{2 \sigma B_{d}+2 \sigma B_{t}+2 B_{d} B_{t}}{\sigma B_{t}}$. Plugging $P_{d}, P_{t}$ into the profit function, we can obtain $\pi^{*}$.

In the second case, $\lambda_{1}=0, \lambda_{2}=0, \lambda_{3} \neq 0$. By $\frac{\partial L}{\partial P_{d}}=0, \frac{\partial L}{\partial P_{t}}=0, \frac{\partial L}{\partial \lambda_{3}}=0$, we obtain that $P_{t}=\frac{A_{t}}{B_{t}}, P_{d}=\frac{2 \sigma A_{t}+A_{d} B_{t}}{2\left(\sigma+B_{d}\right) B_{t}}$. By $\lambda_{3} \geq 0$, we obtain the condition $\mathrm{m} \geq \frac{2\left(\sigma B_{d}+\sigma B_{t}+B_{d} B_{t}\right)}{\sigma B_{t}}$. Plugging $P_{d}, P_{t}$ into the profit function, we can obtain $\pi^{*}$.

In the third case, $\lambda_{1}=0, \lambda_{2} \neq 0, \lambda_{3}=0$. By $\frac{\partial L}{\partial P_{d}}=0, \frac{\partial L}{\partial P_{t}}=0, \frac{\partial L}{\partial \lambda_{2}}=0$, we obtain that $\lambda_{2}=-\frac{\sigma A_{d} B_{t}+2 \sigma A_{t} B_{t}+2 A_{t} B_{d} B_{t}}{\sigma^{2} B_{d}+2 \sigma^{2} B_{t}+2 \sigma B_{d} B_{t}+\sigma B_{t}^{2}+B_{d} B_{t}^{2}}<$ 0 . Thus the third case is infeasible.

In the fourth case, $\lambda_{1}=0, \lambda_{2} \neq 0, \lambda_{3} \neq 0$. By $\frac{\partial L}{\partial P_{d}}=0, \frac{\partial L}{\partial P_{t}}=0, \frac{\partial L}{\partial \lambda_{2}}=0, \frac{\partial L}{\partial \lambda_{3}}=0$, we obtain that $P_{t}=\frac{A_{t}}{B_{t}}, P_{d}=\frac{A_{t}}{B_{t}}, \lambda_{2}=$ $\frac{-2 A_{t} B_{d}+A_{d} B_{t}}{\sigma B_{t}}, \lambda_{3}=-\frac{2 \sigma A_{t} B_{d}-\sigma A_{d} B_{t}+2 \sigma A_{t} B_{t}+2 A_{t} B_{d} B_{t}-A_{d} B_{t}^{2}}{\sigma B_{t}^{2}}$. The condition $\lambda_{2} \geq 0$ and $\lambda_{3} \geq 0$ cannot be satisfied simultaneously and thus the fourth case is infeasible.

In the fifth case, $\lambda_{1} \neq 0, \lambda_{2}=0, \lambda_{3}=0$. By $\frac{\partial L}{\partial P_{d}}=0, \frac{\partial L}{\partial P_{t}}=0, \frac{\partial L}{\partial \lambda_{1}}=0$, we obtain that $\lambda_{1}=-\frac{A_{d}}{\sigma+B_{d}}<0$. Thus the fifth case is infeasible.

In the sixth case, $\lambda_{1} \neq 0, \lambda_{2}=0, \lambda_{3} \neq 0$. By $\frac{\partial L}{\partial P_{d}}=0, \frac{\partial L}{\partial P_{t}}=0, \frac{\partial L}{\partial \lambda_{1}}=0, \frac{\partial L}{\partial \lambda_{3}}=0$, we obtain that $\lambda_{1}=-\frac{A_{d}}{\sigma+B_{d}}<0$. Thus the sixth case is infeasible.

In the seventh case, $\lambda_{1} \neq 0, \lambda_{2} \neq 0, \lambda_{3}=0$. By $\frac{\partial L}{\partial P_{d}}=0, \frac{\partial L}{\partial P_{t}}=0, \frac{\partial L}{\partial \lambda_{1}}=0, \frac{\partial L}{\partial \lambda_{2}}=0$, we obtain that $\lambda_{1}=$ $-\frac{\sigma^{2} A_{d} B_{d}+3 \sigma^{2} A_{d} B_{t}+2 \sigma^{2} A_{t} B_{t}+2 \sigma A_{d} B_{d} B_{t}+2 \sigma A_{t} B_{d} B_{t}+\sigma A_{d} B_{t}^{2}+A_{d} B_{d} B_{t}^{2}}{\left(\sigma B_{d}+\sigma B_{t}+B_{d} B_{t}\right)^{2}}<0$. Therefore, the seventh case is infeasible.

In the eighth case, $\lambda_{1} \neq 0, \lambda_{2} \neq 0, \lambda_{3} \neq 0$. By $\frac{\partial L}{\partial P_{d}}=0, \frac{\partial L}{\partial P_{t}}=0, \frac{\partial L}{\partial \lambda_{1}}=0, \frac{\partial L}{\partial \lambda_{2}}=0, \frac{\partial L}{\partial \lambda_{3}}=0$, we obtain no solutions. 
In sum, we obtain the optimal solution as given in Lemma 2.

\section{Proof of Lemma 3}

To solve the publisher's decision problem under the strategy 3, we write the Lagrange equation as follows. $L=\left(P_{d} * Q_{f 2}+P_{d} * Q_{d}+P_{t} * Q_{t}+\lambda_{1}\left(A_{d}-B_{d} * P_{d}+\sigma\left(P_{t}-P_{d}\right)\right)+\lambda_{2}\left(A_{t}-B_{t} * P_{t}+\sigma\left(P_{d}-P_{t}\right)\right)+\lambda_{3}\left(A_{d}-B_{d} * P_{d}\right)\right.$

There are eight cases to discuss depending on the values of $\lambda_{1}, \lambda_{2}$ and $\lambda_{3}$.

In the first case, $\lambda_{1}=0, \lambda_{2}=0, \lambda_{3}=0$. By $\frac{\partial L}{\partial P_{d}}=0, \frac{\partial L}{\partial P_{t}}=0$, we obtain that $P_{t}=\frac{2 \sigma A_{d}+\sigma A_{t}+2 A_{t} B_{d}}{2\left(2 \sigma B_{d}+\sigma B_{t}+2 B_{d} B_{t}\right)}, P_{d}=\frac{2 \sigma A_{d}+\sigma A_{t}+2 A_{d} B_{t}}{2\left(2 \sigma B_{d}+\sigma B_{t}+2 B_{d} B_{t}\right)}$. Plugging $P_{d}, P_{t}$ into the demand function, we can obtain that $Q_{d} \geq 0, Q_{t} \geq 0$. Plugging $P_{d}, P_{t}$ into the constraints $Q_{f} \geq 0$ and let $m=A_{d} / A_{t}$, we obtain the condition $m>\frac{\sigma B_{d}}{2 \sigma B_{d}+2 \sigma B_{t}+2 B_{d} B_{t}}$. Plugging $P_{d}, P_{t}$ into the profit function, we can obtain $\pi^{*}$.

In the second case, $\lambda_{1}=0, \lambda_{2}=0, \lambda_{3} \neq 0$. By $\frac{\partial L}{\partial P_{d}}=0, \frac{\partial L}{\partial P_{t}}=0, \frac{\partial L}{\partial \lambda_{3}}=0$, we obtain that $P_{t}=\frac{2 \sigma A_{d}+A_{t} B_{d}}{2 B_{d}\left(\sigma+B_{t}\right)}, P_{d}=\frac{A_{d}}{B_{d}}$. By $\lambda_{3} \geq$ 0 , we derive the condition $0<m<\frac{\sigma B_{d}}{2 \sigma B_{d}+2 \sigma B_{t}+2 B_{d} B_{t}}$. Plugging $P_{d}, P_{t}$ into the profit function, we can obtain $\pi^{*}$.

In the third case, $\lambda_{1}=0, \lambda_{2} \neq 0, \lambda_{3}=0$. By $\frac{\partial L}{\partial P_{d}}=0, \frac{\partial L}{\partial P_{t}}=0, \frac{\partial L}{\partial \lambda_{2}}=0$, we obtain that $\lambda_{2}=-\frac{A_{t}}{\sigma+B_{t}}<0$. Thus, the third case is infeasible.

In the fourth case, $\lambda_{1}=0, \lambda_{2} \neq 0, \lambda_{3} \neq 0$. By $\frac{\partial L}{\partial P_{d}}=0, \frac{\partial L}{\partial P_{t}}=0, \frac{\partial L}{\partial \lambda_{2}}=0, \frac{\partial L}{\partial \lambda_{3}}=0$, we obtain that $\lambda_{2}=-\frac{A_{t}}{\sigma+B_{t}}<0$. Therefore, the fourth case is infeasible.

In the fifth case, $\lambda_{1} \neq 0, \lambda_{2}=0, \lambda_{3}=0$. By $\frac{\partial L}{\partial P_{d}}=0, \frac{\partial L}{\partial P_{t}}=0, \frac{\partial L}{\partial \lambda_{1}}=0$, we obtain that $\lambda_{1}=-\frac{2 \sigma A_{d} B_{d}+\sigma A_{t} B_{d}+2 A_{d} B_{d} B_{t}}{2 \sigma^{2} B_{d}+\sigma B_{d}^{2}+\sigma^{2} B_{t}+2 \sigma B_{d} B_{t}+B_{d}^{2} B_{t}}<$ 0 . Thus, the fifth case is infeasible.

In the sixth case, $\lambda_{1} \neq 0, \lambda_{2}=0, \lambda_{3} \neq 0$. By $\frac{\partial L}{\partial P_{d}}=0, \frac{\partial L}{\partial P_{t}}=0, \frac{\partial L}{\partial \lambda_{1}}=0, \frac{\partial L}{\partial \lambda_{3}}=0$, we obtain that $\lambda_{3}=$ $\frac{\sigma A_{t} B_{d}+A_{t} B_{d}^{2}-2 \sigma A_{d} B_{t}-2 A_{d} B_{d} B_{t}-2 \sigma A_{d} B_{d}}{\sigma B_{d}^{2}}$ and $\lambda_{1}=\frac{2 A_{d} B_{t}-A_{t} B_{d}}{\sigma B_{d}}$. The condition $\lambda_{1} \geq 0$ and $\lambda_{3} \geq 0$ cannot be satisfied simultaneously, and thus the sixth case is infeasible.

In the seventh case, $\lambda_{1} \neq 0, \lambda_{2} \neq 0, \lambda_{3}=0$. By $\frac{\partial L}{\partial P_{d}}=0, \frac{\partial L}{\partial P_{t}}=0, \frac{\partial L}{\partial \lambda_{1}}=0, \frac{\partial L}{\partial \lambda_{2}}=0$, we obtain that $\lambda_{1}=$ $-\frac{2 \sigma^{2} A_{d} B_{d}+3 \sigma^{2} A_{t} B_{d}+\sigma^{2} A_{t} B_{t}+4 \sigma A_{d} B_{d} B_{t}+3 \sigma A_{t} B_{d} B_{t}+2 A_{d} B_{d} B_{t}^{2}}{\left(\sigma B_{d}+\sigma B_{t}+B_{d} B_{t}\right)^{2}}<0$. Thus the seventh case is infeasible.

In the eighth case, $\lambda_{1} \neq 0, \lambda_{2} \neq 0, \lambda_{3} \neq 0$. By $\frac{\partial L}{\partial P_{d}}=0, \frac{\partial L}{\partial P_{t}}=0, \frac{\partial L}{\partial \lambda_{1}}=0, \frac{\partial L}{\partial \lambda_{2}}=0, \frac{\partial L}{\partial \lambda_{3}}=0$, we obtain no solutions.

In sum, we obtain solutions as given by Lemma 3 .

\section{Proof of Proposition 1}

We first compare $\pi_{a 1}$ and $\pi_{a 2}$. Note that $B_{t}=1, B_{d}=1$ and $m=A_{d} / A_{t}$.

When $0<m<\frac{2 \sigma B_{d}+2 \sigma B_{t}+2 \sigma B_{d} B_{t}}{\sigma B_{t}}=\frac{2+4 \sigma}{\sigma} \quad, \quad$ we have that $\pi_{a 1}-\pi_{a 2}=\frac{2 \sigma A_{d} A_{t}+A_{t}^{2}\left(\sigma+B_{d}\right)+A_{d}^{2}\left(\sigma+B_{t}\right)}{2\left(\sigma B_{t}+B_{d}\left(\sigma+B_{t}\right)\right)}-$ $\frac{4 \sigma A_{d} A_{t}+4 A_{t}^{2}\left(\sigma+B_{d}\right)+A_{d}^{2}\left(\sigma+2 B_{t}\right)}{8 \sigma B_{t}+4 B_{d}\left(\sigma+2 B_{t}\right)}=\left(2+5 \sigma+4 \sigma^{2}\right) m^{2}+4 \sigma(1+\sigma) m-2 \sigma(1+\sigma)$. It can be obtained that when $m>$ $-\frac{2\left(\sigma+\sigma^{2}\right)}{2+5 \sigma+4 \sigma^{2}}+\sqrt{2} \sqrt{\frac{2 \sigma+9 \sigma^{2}+13 \sigma^{3}+6 \sigma^{4}}{\left(2+5 \sigma+4 \sigma^{2}\right)^{2}}}, \pi_{a 1}-\pi_{a 2}>0$, i.e., profit of strategy 1 is higher than that of strategy 2 . When $0<$ $m<-\frac{2\left(\sigma+\sigma^{2}\right)}{2+5 \sigma+4 \sigma^{2}}+\sqrt{2} \sqrt{\frac{2 \sigma+9 \sigma^{2}+13 \sigma^{3}+6 \sigma^{4}}{\left(2+5 \sigma+4 \sigma^{2}\right)^{2}}}, \pi_{a 1}-\pi_{a 2}<0$, i.e., profit of strategy 1 is lower than that of strategy 2 .

When $m>\frac{2 \sigma B_{d}+2 \sigma B_{t}+2 B_{d} B_{t}}{\sigma B_{t}}=\frac{2+4 \sigma}{\sigma} \quad$ we have $\quad \pi_{a 1}-\pi_{a 2}=\frac{2 \sigma A_{d} A_{t}+A_{t}^{2}\left(\sigma+B_{d}\right)+A_{d}^{2}\left(\sigma+B_{t}\right)}{2\left(\sigma B_{t}+B_{d}\left(\sigma+B_{t}\right)\right)}-\frac{4 \sigma A_{t}^{2} B_{d}+4 \sigma A_{d} A_{t} B_{t}+A_{d}^{2} B_{t}^{2}}{4\left(\sigma+B_{d}\right) B_{t}^{2}}=$ $-\frac{m^{2}-4 \sigma+4 m \sigma}{4+4 \sigma}+\frac{1+\sigma+2 m \sigma+m^{2}(1+\sigma)}{2+4 \sigma}>0$.

Thus, the publisher prefers strategy 1 in this case. 
Combining the above two cases, the publisher prefers strategy 1 when $m>-\frac{2\left(\sigma+\sigma^{2}\right)}{2+5 \sigma+4 \sigma^{2}}+\sqrt{2} \sqrt{\frac{2 \sigma+9 \sigma^{2}+13 \sigma^{3}+6 \sigma^{4}}{\left(2+5 \sigma+4 \sigma^{2}\right)^{2}}}$, and strategy 2 when $0<m<-\frac{2\left(\sigma+\sigma^{2}\right)}{2+5 \sigma+4 \sigma^{2}}+\sqrt{2} \sqrt{\frac{2 \sigma+9 \sigma^{2}+13 \sigma^{3}+6 \sigma^{4}}{\left(2+5 \sigma+4 \sigma^{2}\right)^{2}}}$.

Secondly, we compare $\pi_{a 1}$ and $\pi_{a 3}$. when $0<m<\frac{\sigma B_{d}}{2 \sigma B_{d}+2 \sigma B_{t}+2 \sigma B_{d} B_{t}}=\frac{\sigma}{2+4 \sigma}$, we have $\pi_{a 1}-\pi_{a 3}=$ $\frac{2 \sigma A_{d} A_{t}+A_{t}^{2}\left(\sigma+B_{d}\right)+A_{d}^{2}\left(\sigma+B_{t}\right)}{2\left(\sigma B_{t}+B_{d}\left(\sigma+B_{t}\right)\right)}-\frac{4 \sigma A_{d} A_{t} B_{d}+A_{t}^{2} B_{d}^{2}-4 \sigma A_{d}^{2} B_{t}}{4 B_{d}^{2}\left(\sigma+B_{t}\right)}=\frac{1+2 \sigma+2 \sigma^{2}-4 m \sigma^{2}+2 m^{2}\left(1+4 \sigma+5 \sigma^{2}\right)}{4(1+\sigma)(1+2 \sigma)}>0$. Thus, the publisher prefers strategy 1.

When $m>\frac{\sigma B_{d}}{2 \sigma B_{d}+2 \sigma B_{t}+2 \sigma B_{d} B_{t}}=\frac{\sigma}{2+4 \sigma}$, we have $\pi_{a 1}-\pi_{a 3}=\frac{2 \sigma A_{d} A_{t}+A_{t}^{2}\left(\sigma+B_{d}\right)+A_{d}^{2}\left(\sigma+B_{t}\right)}{2\left(\sigma B_{t}+B_{d}\left(\sigma+B_{t}\right)\right)}-\frac{4 \sigma A_{d} A_{t}+A_{t}^{2}\left(\sigma+2 B_{d}\right)+4 A_{d}^{2}\left(\sigma+B_{t}\right)}{4 \sigma B_{t}+8 B_{d}\left(\sigma+B_{t}\right)}=$ $\frac{2+\left(5+4 m-2 m^{2}\right) \sigma+\left(4+4 m-2 m^{2}\right) \sigma^{2}}{4\left(2+7 \sigma+6 \sigma^{2}\right)}$. When $m>1+\frac{\sqrt{2+7 \sigma+6 \sigma^{2} / \sigma(1+\sigma)}}{\sqrt{2}}, \pi_{a 1}-\pi_{a 3}<0$ and the publisher prefers to delay the physical book. When $m<1+\frac{\sqrt{2+7 \sigma+6 \sigma^{2} / \sigma(1+\sigma)}}{\sqrt{2}}, \pi_{a 1}-\pi_{a 3}>0$ and the publisher prefers strategy 1 .

The above two cases indicate that the strategy 1 is preferred when $m<1+\frac{\sqrt{2+7 \sigma+6 \sigma^{2} / \sigma(1+\sigma)}}{\sqrt{2}}$ and stratey 3 is better when $m>1+\frac{\sqrt{2+7 \sigma+6 \sigma^{2} / \sigma(1+\sigma)}}{\sqrt{2}}$.

[1] In sum, comparing $\pi_{a 1}, \pi_{a 2}$ and $\pi_{a 3}$ we obtain Proposition 1. 